Commun. Fac. Sci. Univ. Ank. Series $A_{1}$

V. 43. pp. 1-11 (1994)

\title{
ON THE FOCAL SURFACES OF THE CONGRUENCES GENERATED BY THE INSTANTANEOUS SCREWING AXES CONNECTED WITH SOME SURFACES
}

TÜRKAN GÙLL

Istanbul Technical Univ. Faculty of Art and Sci. ISTANBUL

(Received July 13, 1993; Accepted Feb. 15, 1994)

\section{ABSTRACT}

In this paper, the focal surfaces of the congruences derived in [1] and [3] have been investigated and correspondences between them have been explained.

\section{INTRODUCTION}

Let a surface $\vec{x}$ be referred to its lines of curvatures. The congruences generated by the instantaneous screwing axes $\vec{G}, \vec{G}$ of the moving trihedrons connected with these lines are respectively,

$$
\left\{\begin{array}{l}
\vec{y}=\vec{r}+\overrightarrow{t g}, \quad \vec{r}=\vec{x}+\frac{1}{r} \vec{\xi} \\
\vec{y}=\vec{r}+\overrightarrow{t g}, \quad \vec{r}=\vec{x}+\frac{1}{\vec{r}} \vec{\xi}
\end{array}\right.
$$

[1]. In case $\vec{y}$ and $\stackrel{\vec{y}}{\mathrm{y}}$ are normal congruences, let the surfaces generating these, be $\vec{z}$ and $\vec{z}$. And let these surfaces be referred to their lines of curvature. The congruence generated by the instantaneous screwing axis $\vec{G}^{*}$ of the moving trihedron connected with the lines of curvature $\mathbf{u}=$ const. of $\overrightarrow{\mathrm{z}}$ are

$$
\stackrel{\rightrightarrows}{\mathbf{y}^{*}}=\mathbf{r}+\overline{\mathbf{t}}^{*} \overrightarrow{\mathbf{g}^{*}}, \quad \overrightarrow{\mathbf{r}}=\overrightarrow{\mathbf{z}}+\frac{1}{\overline{\mathbf{b}}} \overrightarrow{\mathbf{n}}
$$


And the congruence generated by the instanteneous screwing axis $\vec{G} * *$ of the moving trihedron connected with the lines of curvature $\mathrm{v}=$ const. of $\stackrel{\overrightarrow{\mathrm{z}}}{\text { are }}$

$$
\overrightarrow{\mathrm{y}^{* *}}=\overrightarrow{\mathrm{r}}+\overrightarrow{\mathrm{t}} * * \overrightarrow{\mathrm{g}} * *, \overrightarrow{\mathrm{r}}=\overrightarrow{\mathrm{z}}+\frac{1}{\beta} \overrightarrow{\mathrm{n}}
$$

[3].

2. THE PROPERTIES OF THE FOCAL SURFACES OF THE CONGRUENCES $\vec{y}, \vec{y}, \overrightarrow{y^{*}}, \overrightarrow{y^{*}}$

Since $\vec{p}, \vec{k}$ are the focal surfaces of the congruence $\vec{y} ; \vec{p}, \vec{k}$ of $\vec{y}$; $\overrightarrow{p^{*}}, \vec{k} *$ of $\overrightarrow{y^{*}}$; and $\overrightarrow{p^{* *}}, \overrightarrow{k^{*}}$ of $\overrightarrow{y^{*}}[3]$, to investigate considering the cases where they coincide and refer to their lines of curvature, first we may write the moving trihedrons (DARBOUX's trihedrons) connected with a common point before calculating their first and second fundamental forms.

1) Since the moving trihedron connected with the point $\vec{x}$ of the line of curvature $v=$ const. on the surface $\vec{x}(u, v)$ is $\left(\vec{x}_{1}, \vec{x}_{2}, \vec{\xi}\right)$, the trihedrons connected with the focal points corresponding to $\rho_{\text {II }}$ of the focal surfaces $\overrightarrow{\mathrm{p}}, \overrightarrow{\mathrm{p}} * \overrightarrow{\mathrm{k}^{*}}$ belonging to the congruences $\overrightarrow{\mathrm{y}}, \overrightarrow{\mathrm{y}^{*}, \overrightarrow{\mathrm{y}^{* *}} \text { and }}$ coinciding with the center surface $\vec{r}$ of the surface $\vec{x}$, are respectively,

$$
\left(\vec{\xi}, \vec{x}_{2},-\vec{x}_{1}\right), \quad\left(\vec{\xi}, \vec{x}_{2},-\vec{x}_{1}\right), \quad\left(-\vec{\xi},-\vec{x}_{2},-\vec{x}_{1}\right)
$$

2) Since the moving trihedron connected with the point $\vec{x}$ of the line of curvature $u=$ const. on the surface $\vec{x}(u, v)$ is $\left(\vec{x}_{2},-\overrightarrow{x_{1}}, \vec{\xi}\right)$, the trihedrons connected with the focal points corresponding to $\bar{\rho}_{I I}$

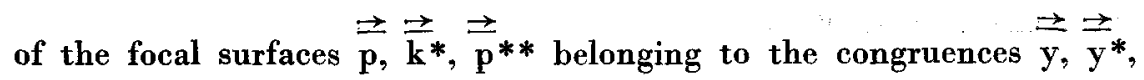
$\overrightarrow{y^{* *}}$ and coinciding with the center surface $\vec{r}$ of the surface $\vec{x}$, are respectively, 


$$
\left(\vec{\xi},-\vec{x}_{1},-\vec{x}_{2}\right), \quad\left(\vec{\xi},-\vec{x}_{1},-\vec{x}_{2}\right), \quad\left(-\vec{\xi}, \vec{x}_{1},-\vec{x}_{2}\right) .
$$

If we calculate the first and the second fundamental forms of the above focal surfaces

1) for the local surfaces $\vec{p}, \overrightarrow{p^{*}}, \overrightarrow{k^{*}} *$, we find,

$$
\left.\begin{array}{l}
\mathrm{E}_{\mathrm{II}}=\overline{\mathrm{E}}^{*} \mathrm{II}=\overline{\mathrm{E}}_{\mathrm{I}}^{* *}=\left(\frac{1}{\mathrm{r}}\right)_{1}^{2} \mathrm{E} \\
\mathrm{F}_{\mathrm{II}}=\overline{\mathrm{F}}_{\mathrm{II}}=\overline{\mathbf{F}}_{\mathrm{I}}^{* *}=\left(\frac{1}{\mathrm{r}}\right)_{1}\left(\frac{1}{\mathrm{r}}\right)_{2} \sqrt{\mathrm{EG}} \\
\mathrm{G}_{\mathrm{II}}=\overline{\mathrm{G}}_{\mathrm{II}}^{*}=\overline{\mathrm{G}}_{\mathrm{I}}^{* *}=\left(\frac{1}{\mathrm{r}}\right)_{2}^{2} \frac{\mathrm{r}^{2}+\mathrm{q}^{2}}{\mathbf{q}^{2}} \mathrm{G},
\end{array}\right\}
$$

$[\mathrm{I}]_{\mathrm{II}}=\left[\mathrm{I}^{*}\right]_{\mathrm{II}}=\left[\mathrm{I}^{* *}\right]_{\mathrm{I}}=\left(\frac{1}{\mathrm{r}}\right)_{1}^{2} \mathbf{E} \mathrm{du}^{2}+2\left(\frac{1}{\mathrm{r}}\right)_{1}\left(\frac{1}{\mathrm{r}}\right) \sqrt{\mathbf{E G}} \mathrm{dudv}$ $+\left(\frac{1}{r}\right)_{2}^{2} \frac{r^{2}+q^{2}}{q^{2}} G d v^{2}$

and

$$
\begin{aligned}
& \mathrm{L}_{\mathrm{II}}=\overline{\mathrm{L}}_{\mathrm{II}}=\overline{\mathrm{L}}^{* *}=\left(\frac{1}{\mathrm{r}}\right)_{1} \text { r E } \\
& \mathrm{M}_{\mathrm{II}}=\overline{\mathbf{M}}^{*}{ }_{\mathrm{II}}=\overline{\mathrm{M}}_{\mathrm{I}}^{* *}=0 \\
& \mathrm{~N}_{\mathrm{II}}=\overline{\mathbf{N}}^{*} \mathrm{II}=\overline{\mathrm{N}}^{* *} \mathrm{II}=\left(\frac{1}{\mathbf{r}}\right)_{2} \frac{\overline{\mathbf{r q}}}{\mathbf{q}} \mathbf{G}
\end{aligned}
$$

$[\mathrm{II}]_{\mathrm{II}}=\left[\bar{\Pi}^{*}\right]_{\mathrm{II}}=\left[\bar{\Pi}^{* *}\right]_{\mathrm{I}}=\mathbf{r}\left[\left(\frac{1}{\mathbf{r}}\right)_{1} \mathbf{E} \mathrm{du}^{2}+\left(\frac{1}{\mathbf{r}}\right)_{2} \frac{\overline{\mathbf{q}}}{\mathbf{q}} \mathbf{G} \mathbf{d v}^{2}\right]$.

From these we may derive the below conclusion:

Conclusion: 2.1. The focal surfaces $\vec{p}, \overrightarrow{p^{*}}, \overrightarrow{k^{* *}}$ of the congruences $\vec{y}, \overrightarrow{y^{*}}, \overrightarrow{y^{* *}}$ are different positions of the center surface $\vec{r}$ of the base surface $\vec{x}$, in space. 
Also, the Gaussian and the mean curvature of these focal surfaces, we find

$$
\mathrm{K}_{\mathrm{II}}=\overline{\mathbf{K}}^{*}{ }_{\mathrm{II}}=\overline{\mathrm{K}}_{\mathrm{I}}^{* *}=\frac{\mathrm{q} \overline{\mathrm{q}}}{\left(\frac{1}{\mathrm{r}}\right)_{1}\left(\frac{1}{\mathrm{r}}\right)_{2}}
$$

and

$$
\mathbf{H}_{\mathrm{II}}={ }^{*} \overline{\mathbf{H}}_{\mathrm{II}}=\mathrm{I}^{*} \overline{\mathbf{H}}^{*}=\frac{\left(\frac{1}{\mathbf{r}}\right)_{1} \mathrm{qq}-\left(\frac{1}{\mathbf{r}}\right)_{2}\left(\mathrm{r}^{2}+\mathbf{q}^{2}\right)}{2 \mathbf{r}\left(\frac{1}{\mathbf{r}}\right)_{1}\left(\frac{1}{\mathbf{r}}\right)_{2}}
$$

Since, $r_{1} \neq 0, r_{2} \neq 0$ from (2.1) and (2.3) we derive the conditions $\mathrm{F}_{\mathrm{II}}=\overline{\mathbf{F}}^{*} \mathrm{II}=\overline{\mathbf{F}}_{\mathrm{I}}^{* *} \neq 0$ and $\mathrm{M}_{\mathrm{II}}=\overline{\mathbf{M}}^{*} \mathrm{II}=\overline{\mathbf{M}}_{\mathrm{I}}^{* *}=0$. From these, the following theorem may be stated:

Theorem 2.2. Since the surface $\vec{x}(u, v)$ cannot be a canal surface or at the same time cannot be both Mulür surface and tube-shaped canal surface, the parameter curves $v=$ const. and $u=$ const. of the focal surfaces $\vec{p}, \vec{p}^{*}, \vec{k}^{* *}$ of the congruences $\vec{y}, \stackrel{\rightrightarrows}{y^{*}, \vec{y}^{*}}$ cannot be the lines of curvature.

Since $\mathrm{q} \neq 0, \overline{\mathrm{q}} \neq 0, \mathrm{r}_{1} \neq 0, \mathrm{r}_{2} \neq 0$ in (2.5) and (2.6), we find $\mathbf{K}_{\mathrm{II}}=\overline{\mathbf{K}}^{*} \mathrm{II}=\overline{\mathbf{K}}_{\mathrm{I}}^{* *} \neq 0$ and $\mathrm{H}_{\mathrm{II}}=\overline{\mathbf{H}}^{*} \mathrm{II}=\overline{\mathbf{H}}_{\mathrm{I}}^{* *} \neq 0$.

Therefore the theorem below may be stated:

Theorem 2.3. Since the surface $\vec{x}(u, v)$ cannot be Mulür surface, canal surface or tube-shaped surface, the focal surfaces $\vec{p}, \vec{p} *, \vec{k}^{* *}$ of the congruences $\vec{y}, \overrightarrow{y^{*}}, \overrightarrow{y^{* *}}$ respectively, cannot be developable surface, minimal surface.

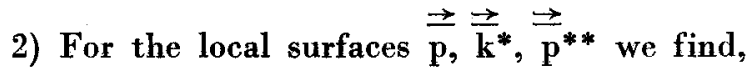




$$
\begin{aligned}
& \overline{\mathrm{E}}_{\mathrm{II}}=\overline{\mathrm{E}}_{\mathrm{I}}^{*}=\overline{\mathrm{E}}^{* *} \mathrm{II}=\left(\frac{1}{\overline{\mathbf{r}}}\right)_{1}^{2} \frac{\overline{\mathbf{r}}^{2}+\overline{\mathbf{q}}^{2}}{\overline{\mathbf{q}}^{2}} \mathrm{E} \\
& \overline{\mathbf{F}}_{\mathrm{II}}=\overline{\mathbf{F}}_{\mathrm{I}}^{*}=\overline{\mathbf{F}}^{* *_{\mathrm{II}}}=\left(\frac{1}{\overline{\mathbf{r}}}\right)_{1}\left(\frac{1}{\overline{\mathbf{r}}}\right)_{2} \sqrt{\overline{\mathrm{EG}}} \\
& \overline{\mathrm{G}}_{\mathrm{II}}=\overline{\mathrm{G}}^{*} \mathrm{I}=\overline{\mathrm{G}}^{* *} \mathrm{II}=\left(\frac{1}{\overline{\mathbf{r}}}\right)_{2}^{2} \mathrm{G} \text {, } \\
& {[\overline{\mathbf{I}}]_{\mathrm{II}}=\left[\overline{\mathbf{I}}^{*}\right]_{\mathrm{I}}=\left[\overline{\mathbf{I}}^{* *}\right]_{\mathrm{II}}=\left(\frac{1}{\overline{\mathbf{r}}}\right)_{1}^{2} \frac{\overline{\mathbf{r}}^{2}+\overline{\mathbf{q}}^{2}}{\overline{\mathbf{q}}^{2}} \mathbf{E ~ d u ^ { 2 } +}} \\
& 2\left(\frac{1}{\overline{\mathbf{r}}}\right)_{1}\left(\frac{1}{\overline{\mathbf{r}}}\right)_{2} \sqrt{ } \mathrm{EG} d \mathrm{du} \mathrm{v}+\left(\frac{1}{\overline{\mathbf{r}}}\right)_{2}^{2} \mathrm{G} d \mathrm{v}^{2}
\end{aligned}
$$

and

$$
\begin{aligned}
& \overline{\mathbf{L}}_{\mathrm{II}}=\overline{\mathbf{L}}^{*} \mathrm{I}=\overline{\mathbf{L}}_{\mathrm{II}}^{* *}=\left(\frac{1}{\overline{\mathbf{r}}}\right)_{1} \frac{\overline{\mathrm{rq}}}{\overline{\mathrm{q}}} \mathrm{E} \\
& \overline{\mathbf{M}}_{\mathrm{II}}=\overline{\mathbf{M}}_{\mathrm{I}}^{*}=\overline{\mathbf{M}}_{\mathrm{I}}^{* *}=0 \\
& \overline{\mathbf{N}}_{\mathrm{II}}=\overline{\mathbf{N}}_{\mathrm{I}}^{*}=\overline{\mathbf{N}}^{* *} \mathrm{II}=\left(\frac{1}{\overline{\mathbf{r}}}\right)_{2} \overline{\mathbf{r}} \mathrm{G}
\end{aligned}
$$

$[\overline{\Pi \Pi}]_{\mathrm{II}}=\left[\bar{\Pi}^{*}\right]_{\mathrm{I}}=\left[\bar{\Pi}^{* *}\right]_{\mathrm{II}}=\dot{\mathbf{r}}\left[\left(\frac{1}{\mathbf{r}}\right)_{1} \frac{\mathrm{q}}{\overline{\mathrm{I}}} \mathrm{E} \mathrm{du}^{2}+\left(\frac{1}{\overline{\mathbf{r}}}\right)_{2} \mathbf{G} \mathbf{d v}\right]$

From these we may write the below conclusion:

Conclusion 2.4. The focal surfaces $\vec{p}, \overrightarrow{k^{*}}, \vec{p}^{* *}$ of the congruences $\vec{y}, \overrightarrow{y^{*}}, \overrightarrow{y^{* *}}$ respectively, are different positions of the center surface $\vec{r}$ of the base surface $\vec{x}$, in space.

Also, the values of $K$ and $H$ for these focal surfaces are found as

$$
\overline{\mathbf{K}}_{\mathrm{II}}=\overline{\mathbf{K}}_{\mathrm{I}}^{*}=\overline{\mathbf{K}}_{\mathrm{II}}^{* *}=\frac{\overline{\mathrm{q} \overline{\mathrm{q}}}}{\left(\frac{1}{\overline{\mathbf{r}}}\right)_{1}\left(\frac{\mathbf{1}}{\overline{\mathbf{r}}}\right)_{2}}
$$


and

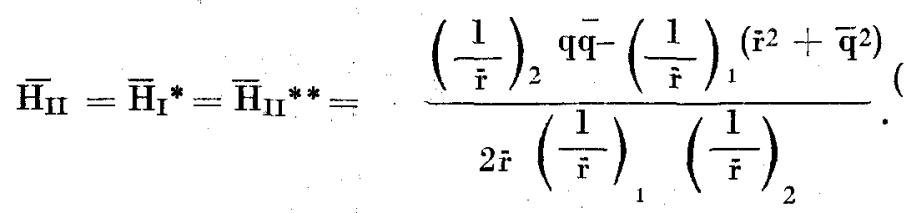

Since $\overline{\mathbf{r}}_{1} \neq 0, \overline{\mathbf{r}} \neq 0$ from (2.7) and (2.9), we find the conditions $\overline{\mathbf{F}}_{\mathrm{II}}=\overline{\mathrm{F}}_{\mathrm{I}}^{*}=\overline{\mathrm{F}}_{\mathrm{II}}^{* *} \neq 0$ and $\overline{\mathrm{M}}^{*} \mathrm{II}=\overline{\mathrm{M}}_{\mathrm{I}}^{*}=\overline{\mathrm{M}}_{\mathrm{II}}^{* *}=0$.

From these we may write the below theorem:

Theorem 2.5. Since the surface $\vec{x}(u, v)$ cannot be canal surface or tube-shaped surface, the parameter curves $v=$ const. and $\mathbf{u}=$

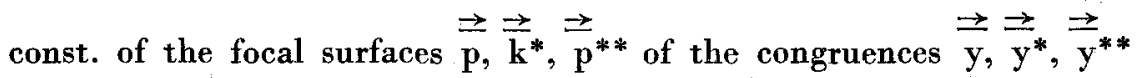
cannot be lines of curvature.

Since $q \neq 0, \vec{q} \neq, 0 \overline{\mathbf{r}}_{1} \neq 0, \overline{\mathrm{r}}_{2} \neq 0$ in (2.11) and (2.12), therefore the below theorem may be written.

Theorem 2.6. Since the surface $\vec{x}(u, v)$ cannot be Mulür surface or general cylindric surface, canal surface or tub-shaped surface, the

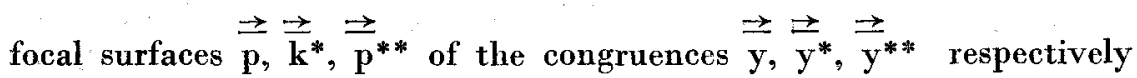
cannot be developable surface, minimal surface.

On the other hand to investigate the focal surface $\vec{k}$ and $\vec{k}$ belonging to the congruences $\vec{y}$ and $\vec{y}$ respectively and coinciding with the center surface of the surfaces $\vec{z}$ and $\stackrel{\vec{z}}{\vec{b}}$ but which do not coincide with the center surfaces $\vec{r}$ and $\frac{\vec{r}}{r}$ of the surface $\vec{x}(u, v)$, first, we may write the moving trihedrons connected with the focal point corresponding to $\rho_{1}$ of $\vec{k}$, connected with the focal point corresponding to $\bar{p}_{I}$ of $\vec{k}$ before calculating their first and second fundamental forms. 
1) For the focal surface $\vec{k}$, from

$$
\vec{k}_{1}=-\left(\frac{1}{b}\right)_{1} \vec{g}, \vec{k}_{2}=\frac{r_{2} \vec{q}}{r_{1} q-q_{1} \mathbf{r}} \vec{x}_{1}-\left(\frac{1}{b}\right)_{2} \vec{g}
$$

and

$$
\begin{aligned}
& \overrightarrow{\mathbf{n}}_{\mathrm{I}}=\frac{\overrightarrow{\mathbf{k}}_{1} \Lambda \overrightarrow{\mathbf{k}}_{2}}{\sqrt{{\overrightarrow{\left(\mathbf{k}_{1}\right.}}_{\left.\mathbf{k}_{2}\right)}}}=\frac{\overrightarrow{q \mathbf{x}_{2}-\mathbf{r} \vec{\xi}}}{\sqrt{\mathbf{r}^{2}+\mathrm{q}^{2}}}=-\overrightarrow{\mathrm{z}}_{\mathbf{1}}^{-}, \\
& \overrightarrow{\mathbf{n}}_{\mathrm{I}} \Lambda(-\overrightarrow{\mathrm{g}})=\overrightarrow{\mathrm{x}}_{1}
\end{aligned}
$$

the trihedron

$$
\left(-\vec{g}, \vec{x}_{1}, \vec{n}_{1}\right)
$$

is found.

2) For the focal surface $\overrightarrow{\vec{k}}$, from

$$
\overrightarrow{\mathbf{k}_{1}}=-\left(\frac{1}{\bar{\beta}}\right)_{1} \overrightarrow{\mathrm{g}}-\frac{\overline{\mathrm{r}}_{2} \mathrm{q}}{\overline{\mathbf{q}}_{2} \overline{\mathbf{r}}-\overline{\mathbf{r}}_{2} \overline{\mathrm{q}}} \overrightarrow{\mathbf{x}_{2}}, \overrightarrow{\mathbf{k}_{2}}=-\left(\frac{1}{\bar{\beta}}\right)_{2} \overrightarrow{\mathrm{g}}
$$

and

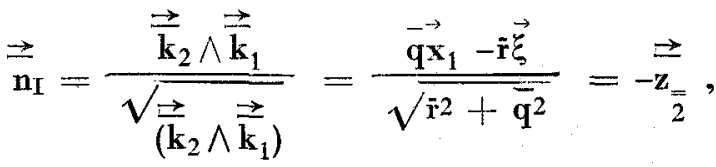

$$
\begin{aligned}
& \overrightarrow{\mathbf{n}_{\mathrm{I}}} \wedge \underset{(-\mathrm{g})}{\overrightarrow{\mathrm{g}}}=\overrightarrow{\mathbf{x}_{2}}
\end{aligned}
$$

the trihedron is

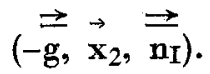

If we calculate the first and the second fundamental forms of the focal surfaces 
1) For $\vec{k}$, we find,

$$
\begin{aligned}
& \mathrm{E}_{\mathrm{I}}=\left(\frac{1}{\mathrm{~b}}\right)_{1}^{2} \mathrm{E} \\
& \mathrm{F}_{\mathrm{I}}=\left(\frac{1}{\mathrm{~b}}\right)_{1}\left(\frac{1}{\mathrm{~b}}\right)_{2} \sqrt{\mathrm{EG}} \\
& \mathrm{G}_{\mathrm{I}}=\left[\left(\frac{\mathrm{r}_{2} \overline{\mathrm{q}}}{\mathrm{r}_{1} \mathrm{q}^{-\mathrm{q}_{1} \mathbf{r}}}\right)^{2}+\left(\frac{1}{\mathrm{~b}}\right)_{2}^{2}\right] \mathrm{G},
\end{aligned}
$$

$[\mathrm{I}]_{\mathrm{I}}=\left(\frac{1}{\mathrm{~b}}\right)_{1}^{2} \mathrm{E} \mathrm{du}^{2}+2\left(\frac{1}{\mathrm{~b}}\right)_{1}\left(\frac{1}{\mathrm{~b}}\right)_{2} \sqrt{\mathrm{EG}} \mathrm{dudv}+$

$\left[\left(\frac{r_{1} \bar{q}}{r_{1} q-q_{1} r}\right)^{2}+\left(\frac{1}{b}\right)_{2}^{2}\right] G d^{2}$

and

$$
\begin{gathered}
\mathrm{L}_{\mathrm{I}}=-\left(\frac{1}{\mathrm{~b}}\right)_{1} \frac{\mathrm{r}_{1} \mathrm{q}-\mathrm{q}_{1} \mathrm{r}}{\mathrm{r}^{2}+\mathrm{q}^{2}} \mathrm{E} \\
\mathrm{M}_{\mathrm{I}}=0 \\
\mathrm{~N}_{\mathrm{I}}=\left(\frac{1}{\overline{\mathrm{b}}}\right)_{1} \frac{\mathrm{r}^{2} \overline{\mathrm{q}}^{2}}{\mathrm{r}_{1} \mathrm{q}-\mathrm{q}_{1} \mathrm{r}} \mathrm{G} \\
{[\mathrm{II}]_{\mathrm{I}}=-\left(\frac{1}{\mathrm{~b}}\right)_{1} \frac{\mathrm{r}_{1} \mathrm{q}-\mathrm{q}_{1} \mathbf{r}}{\mathbf{r}^{2}+\mathrm{q}^{2}} \mathrm{E} \mathbf{d u}^{2}+\left(\frac{1}{\overline{\mathrm{b}}}\right)_{1} \frac{\mathbf{r}^{2} \overline{\mathbf{q}}^{2}}{\mathrm{r}_{1} \mathrm{q}-\mathrm{q}_{1} \mathbf{r}} \mathrm{G} \mathbf{d \mathbf { v } ^ { 2 }}}
\end{gathered}
$$

and also,

$$
\begin{aligned}
& K_{I}=-\frac{q^{2}\left(r_{1} q-q_{1} r\right)^{2}}{\left(\frac{1}{b}\right)_{1}\left(\frac{1}{\bar{b}}\right)_{1} r^{2}\left(r^{2}+q^{2}\right)^{2}},
\end{aligned}
$$

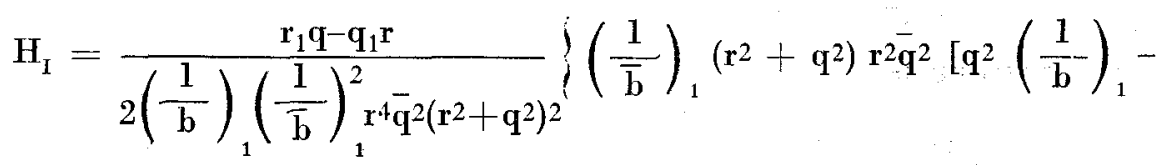

$$
\begin{aligned}
& \left.\left.-\mathbf{r}^{2}\left(\frac{1}{\bar{b}}\right)_{1}\right]-\left(\frac{1}{\bar{b}}\right)_{2}^{2}\left(r_{1} q_{-} q_{1} r\right) q^{2}\right)
\end{aligned}
$$


2) For $\overrightarrow{\mathrm{k}}$,

$$
\begin{aligned}
& \overline{\mathbf{E}}_{\mathrm{I}}=\left[\left(\frac{1}{\bar{\beta}}\right)_{1}^{2}+\left(\frac{\overline{\mathbf{r}}_{2 \mathrm{q}}}{\overline{\mathbf{q}}_{2} \overline{\mathbf{r}}-\overline{\mathbf{r}}_{2} \bar{q}}\right)^{2}\right] \mathbf{E} \\
& \overline{\mathbf{F}}_{\mathrm{I}}=\left(\frac{1}{\bar{\beta}}\right)_{1}\left(\frac{1}{\bar{\beta}}\right)_{2} \sqrt{\mathbf{E G}} \\
& \overline{\mathbf{G}}_{\mathrm{I}}=\left(\frac{1}{\bar{\beta}}\right)_{2}^{2} \mathrm{G}
\end{aligned}
$$

$$
\begin{aligned}
{[\mathbf{I}]_{\mathrm{I}}=\left[\left(\frac{1}{\bar{\beta}}\right)_{1}^{2}+\left(\frac{\overline{\mathbf{r}}_{2} \mathrm{q}}{\overline{\bar{q}}_{2} \overline{\mathrm{r}}-\overline{\mathbf{r}}_{2} \bar{q}}\right)^{2}\right] \mathrm{E} \mathrm{du}+2\left(\frac{1}{\bar{\beta}}\right)_{1}\left(\frac{1}{\bar{\beta}}\right)_{2} \sqrt{\mathrm{EG}} \mathrm{du} \mathrm{dv} } \\
+\left(\frac{1}{\bar{\beta}}\right)_{2}^{2} \mathrm{G} \mathrm{dv2}
\end{aligned}
$$

and

$$
\begin{aligned}
& \overline{\mathrm{L}}_{\mathrm{I}}=\left(\frac{1}{\beta}\right)_{2} \frac{\overline{\mathbf{r}}_{2} \mathbf{q}^{2}}{\overline{\mathbf{q}}_{2} \overline{\mathbf{r}}^{-} \overline{\mathbf{r}}_{2} \bar{q}} \mathbf{E} \\
& \overline{\mathrm{M}}_{\mathrm{I}}=0 \\
& \overline{\mathbf{N}}_{\mathbf{l}}=\left(\frac{1}{\bar{\beta}}\right)_{2} \frac{\overline{\mathbf{r}}_{2} \overline{\mathbf{q}}-\overline{\mathbf{q}}_{2} \overline{\mathbf{r}}}{\overline{\mathbf{r}}^{2}+\overline{\mathbf{q}}^{2}} \mathbf{G}, \\
& {[\overline{\mathrm{II}}]_{\mathrm{I}}=\left(\frac{1}{\beta}\right) \frac{\overline{\mathbf{r}}^{2} \mathbf{q}^{2}}{\overline{\mathbf{q}}_{2} \overline{\mathbf{r}}-\overline{\mathbf{r}}_{2} \overline{\mathbf{q}}} \mathbf{E} \mathbf{d u}^{2}+\left(\frac{1}{\bar{\beta}}\right)_{2} \frac{\overline{\mathbf{r}}_{2} \overline{\mathbf{q}}-\overline{\mathbf{q}_{2}} \overline{\mathbf{r}}}{\overline{\mathbf{r}}_{2}+\overline{\mathbf{q}}^{2}} \mathbf{G} \mathbf{v} \mathbf{d}^{2}}
\end{aligned}
$$

and also,

$$
\begin{aligned}
& \overline{\mathbf{K}}_{\mathrm{I}}=-\frac{\overline{\mathbf{q}}^{2}\left(\overline{\mathbf{q}}_{2} \mathbf{\mathbf { r }}-\overline{\mathbf{q}}_{2} \overline{\mathbf{r}}\right)^{2}}{\left(\frac{1}{\bar{\beta}}\right)_{2}\left(\frac{1}{\beta}\right)_{2} \overline{\mathbf{r}}^{2}\left(\dot{\mathbf{r}}^{2}+\overline{\mathbf{q}}^{2}\right)^{2}}, \\
& \overline{\mathrm{H}}_{\mathrm{I}}=\frac{\overline{\mathrm{q}}_{2} \overline{\mathrm{r}}_{-\mathrm{r}} \overline{\mathrm{r}}_{2} \overline{\mathrm{q}}}{2\left(\frac{1}{\bar{\beta}}\right)_{2}\left(\frac{1}{\beta}\right)_{2}^{2}\left(\overline{\mathbf{r}}^{2}+\overline{\mathbf{q}}^{2}\right) \overline{\mathbf{r}}^{4} \overline{\mathrm{q}}^{2}}
\end{aligned}
$$


$\left\{\overline{\mathbf{r}}^{2} \mathrm{q}^{2}\left(\overline{\mathbf{r}}^{2}+\overline{\mathbf{q}}^{2}\right)\left(\frac{\mathrm{I}}{\bar{\beta}}\right)_{2}\left[\left(\frac{1}{\beta}\right)_{2} \dot{\mathbf{r}}^{2}-\left(\frac{1}{\bar{\beta}}\right)_{2} \overline{\mathrm{q}}^{2}\right]-\left(\frac{1}{\bar{\beta}}\right)_{2}^{2}\left(\overline{\mathbf{q}} 2 \dot{\mathbf{r}}-\dot{\mathbf{r}}_{2} \overline{\mathrm{q}}\right)^{2} \overline{\mathrm{q}}^{2}\right\}$ are written.

1) Since the focal surface $\vec{k}$ coincides with the center surface belonging to the lines of curvature $v=$ const. of the surface $\vec{z}$, that is $\overrightarrow{\mathbf{k}}=\overrightarrow{\mathbf{r}}+\rho \overrightarrow{\mathrm{g}}=\overrightarrow{\mathrm{z}}+\frac{\mathrm{l}}{\overrightarrow{\mathrm{b}}} \overrightarrow{\mathbf{n}}=\overrightarrow{\mathrm{z}}-\frac{1}{\mathrm{~b}} \overrightarrow{\mathrm{g}}$, we may write,

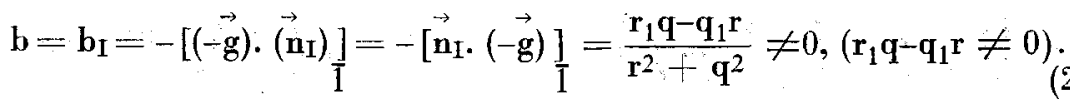

Since $F_{I} \neq 0\left(b_{1} \neq 0, b_{2} \neq 0\right)$ in (2.15) and $M_{I}=0$ in (2.17), the below theorem may be written.

Theorem 2.7. Since the surface $\vec{z}$ cannot be canal surface or tube-shaped surface, the parameter curves $v=$ const. and $u=$ const. of the focal surface $\vec{k}$ of the congruence $\vec{y}$, cannot be lines of curvature.

From (2.19) and $(2.20) \quad \mathrm{K}_{\mathrm{I}} \neq 0, \quad \mathrm{H}_{\mathrm{I}} \neq 0\left(\mathrm{q} \neq 0, \quad \mathbf{r}_{1} \mathrm{q}-\mathrm{q}_{1} \mathbf{r} \neq 0\right)$ are seen. From this the below theorem may be written.

Theorem 2.8. Since the surface $\vec{x}(u, v)$ cannot be Mulür surface and the surface which have the lines of curvature $v=$ const, consisting of plane curves, the focal surface $\vec{k}$ of the congruence $\vec{y}$, cannot be developable surface, minimal surface.

2) Since the focal surface $\vec{k}$ coincides with the central surface belonging to the lines of curvature $u=$ const. on the surface $\stackrel{\vec{z}}{\overrightarrow{t h a t}}$ is $\overrightarrow{\mathbf{k}}=\overrightarrow{\mathbf{r}}+\overrightarrow{\rho \mathrm{g}}=\overrightarrow{\mathrm{z}}+\frac{1}{\bar{\beta}} \overrightarrow{\mathbf{n}}=\overrightarrow{\mathrm{z}}-\frac{1}{\bar{\beta}} \overrightarrow{\mathrm{g}}$, we may write

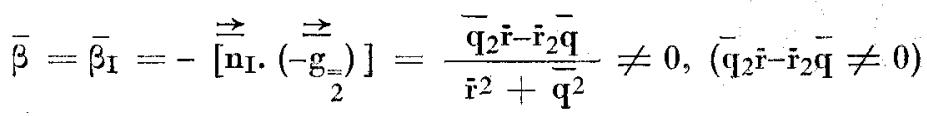


It can be seen that at $(2.21), \overline{\mathrm{F}}_{\mathrm{I}} \neq 0\left(\bar{\beta}_{1} \neq 0, \bar{\beta}_{2} \neq 0\right)$. If we take the condition $\bar{M}_{\mathrm{I}}=0$ at (2.23) into consideration together with these, we may write the below theorem.

Theorem 2.9. Since the surface $\vec{z}$ cannot be canal surface or tube-shaped surface, the parameter curves $\mathrm{v}=$ const. and $\mathbf{u}=$ const. on the focal surface $\vec{k}$ of the congruence $\vec{y}$, cannot be lines of curvature.

From (2.25) and (2.26) $\overline{\mathbf{K}}_{\mathrm{I}} \neq 0, \overline{\mathrm{H}}_{\mathrm{I}} \neq 0 \quad\left(\overline{\mathrm{q}} \neq 0, \quad \overline{\mathrm{q}}_{2} \overline{\mathrm{r}}_{-\dot{\mathbf{r}}_{2} \overline{\mathrm{q}}} \neq 0\right)$ are seen. From this below theorem may be written.

Theorem 2.10. Since the surface $\vec{x}(u, v)$ cannot be Mulür surface and the surface which have with the lines of curvature $u=$ const. consisting of plane curves, the focal surface $\vec{k}$ of the congruence $\vec{y}$ cannot be developable surface, minimal surface.

\section{REFERENCE}

[1] T. GÜL, On the rectilinear congruences generated by the instantaneous screwing axes connected with a surface, Bull. of Techn. Univ. İst. (1990).

[2] T. GUL, On the surfaces which cut ortogonally the congruences generated by the instantaneous screwing axes connected with a surface, Y. Uं.D. İstanbul (1989).

[3] T. GUL, On the congruences of lines generated by the instantaneous screwing axes connected with some surfaces, Communications Seri Al Ankara (1991).

[4] P. EISENHART, A treatige on the Differential Geometry of Curves and Surfaces, New York, (1960). 